Revista de
Economild
Contemporâned

\title{
GASTOS PÚBLICOS E RECEITAS FISCAIS NO BRASIL: UMA ANÁLISE A PARTIR DE SENSIBILIDADE DE PARÂMETROS
}

\author{
Fernando Motta Correia ${ }^{a}$ \\ Gilberto da Silveira Barros Neto ${ }^{b}$ \\ Recebido em 01 maio 2019 \\ Aceito em 13 abril 2021 \\ Received on 1 May 2019 \\ Accepted on 13 April 2021
}

a Professor do Departamento de Economia da Universidade Federal do Paraná (UFPR). Curitiba, PR, Brasil. ORCID: https://orcid.org/0000-0002-0739-6103.

${ }^{\text {b }}$ Doutor pelo Programa de Pós-Graduação em Desenvolvimento Econômico da Universidade Federal do Paraná (PPGDE/UFPR). Curitiba, PR, Brasil. ORCID: https://orcid.org/0000-0002-5246-8575.

RESUMO: Este artigo busca mensurar os multiplicadores fiscais para a economia brasileira, levando em consideração os possíveis efeitos dos choques da atividade econômica sobre as receitas fiscais do governo central, a partir de uma análise de sensibilidade de parâmetros. A pesquisa faz uso de um vetor auto-regressivo estrutural com tratamento dos problemas de quebra estrutural e de identificação do modelo estrutural. $\mathrm{O}$ choque da atividade econômica sobre as receitas do governo central foi usado como referência para a análise de sensibilidade de parâmetros na tentativa de resolver o problema de identificação do modelo estrutural. Os principais resultados mostram que alterações na carga tributária têm papel de destaque na medição dos multiplicadores fiscais.

PALAVRAS-CHAVE: política fiscal; vetor auto-regressivo; Brasil.

CLASSIFICAÇÃO JEL: C32; E32; E62.

Correspondência para: Fernando Motta Correia

Contato: fmcorreia@ufpr.br 


\title{
PUBLIC EXPENDITURES AND TAX REVENUES IN BRAZIL: A PARAMETER SENSITIVITY ANALYSIS
}

\begin{abstract}
This article aims to measure fiscal multipliers for the Brazilian economy, considering the possible effects of economic activity shocks on central government fiscal revenues, based on a sensitivity analysis of parameters. The research was conducted with a structural vector autoregressive model with treatment of the problems of structural breakdown and identification of the structural model, which was addressed using the shock of economic activity on central government revenues as a reference for the parameter sensitivity analysis. The main results show that changes in the tax burden have important effects on the measurement of tax multipliers.
\end{abstract}

KEYWORDS: fiscal policy; vector autoregressive; Brazil. 


\section{INTRODUÇÃO}

Desde o início do processo de estabilização econômica na década de 1990, a carga tributária aumentou consideravelmente - passou de 25\%, antes do Plano Real, para valores superiores a $35 \%$ em relação ao PIB no período recente.

Aliado ao aumento da carga tributária total, o período pós Plano Real assistiu ao aumento da carga tributária nas faixas de renda mais baixas, quando analisada a distribuição da carga tributária bruta por estrato de renda. Enquanto em 2004, para os estratos de renda até dois salários mínimos, a carga tributária total era de 48\%, em 2014 esse valor passou para pouco mais de 53\%. Para os estratos de renda superior a trinta salários mínimos, a carga tributária manteve-se estável em torno de $27 \%$. O tamanho da carga tributária e suas distorções chamam a atenção para a forma como as receitas fiscais reagem frente aos ciclos da atividade econômica.

Em função da natureza do perfil da carga tributária brasileira, seus impactos podem se associar ao tamanho dos multiplicadores fiscais. Uma vez que o crescimento da carga tributária total é acompanhado por uma maior participação das faixas de renda mais baixas, nos momentos de baixo ritmo de crescimento econômico, a perda de receita fiscal é amplificada devido à elevada dependência da estrutura tributária das faixas de renda baixas, uma vez que os estratos salariais inferiores a dois salários mínimos são mais vulneráveis aos momentos de recessão. Particularmente importante para a eficácia da política fiscal é identificar como essa mudança significativa da carga tributária afetou a carga tributária marginal, no sentido da sensibilidade da arrecadação de impostos do governo brasileiro diante de variações no produto.

A literatura associada à medição dos multiplicadores fiscais apresenta resultados conflitantes. Há vários fatores que podem justificar essa evidência: a metodologia, o horizonte temporal da pesquisa, o tipo de economia envolvida na análise etc. A esse respeito, poderíamos comparar Blanchard e Perotti (2002) e Mountford e Uhlig (2009), de modo que os primeiros associam a política de gastos como mais eficaz em afetar a atividade econômica, enquanto Mountford e Uhlig (2009) sustentam que uma política tributária, em termos de velocidade e impacto, teria maior impacto sobre a produção.

Nas últimas décadas, a metodologia associada aos vetores autorregressivos (VAR) tornou-se a principal abordagem na avaliação dos efeitos da política fiscal. A maioria das pesquisas aplicadas ao caso brasileiro utilizam a metodologia dos vetores autorregressivos estruturais (em língua inglesa, structural vector autoregressive - SVAR), o que permite ampliar a credibilidade na estimação dos multiplicadores fiscais. Todavia, a literatura empírica brasileira apresenta algumas limitações, sobretudo no que diz respeito ao tratamento do processo gerador de dados, bem como à etapa de identificação da matriz estrutural do SVAR. Sobre isso, Peres (2006, 2012), Peres e Ellery Jr. (2009) 
e Cavalcanti e Silva (2010) usam a identificação estrutural em modelos autorregressivos com três variáveis, na mesma linha que Blanchard e Perotti (2002).

No caso em particular do procedimento de identificação do modelo estrutural alguns trabalhos levam em consideração as limitações do governo em responder discricionariamente, via receitas fiscais, aos ciclos econômicos. Assim, a necessidade de mensuração dos multiplicadores fiscais deve vir acompanhada por uma restrição no parâmetro que capta o efeito dos choques da atividade econômica sobre as receitas fiscais do governo. Poucos trabalhos aplicados ao caso brasileiro fazem uso do procedimento de restrição de parâmetros, porém, não desenvolvem uma análise de sensibilidade para o parâmetro restrito como em Blanchard e Perotti (2002) e Peres (2006). A ausência de pesquisas com esse tipo de procedimento é particularmente interessante, haja vista os possíveis efeitos das mudanças na base tributária que se observa desde o início da fase de estabilização econômica na década de 1990. Além disso, no âmbito da contabilidade pública, a previsão do efeito dos ciclos econômicos sobre variáveis fiscais como gastos públicos e tributos auxiliam no processo de elaboração e planejamento orçamentário público.

O presente artigo mensura os impactos das variáveis fiscais (gastos do governo e receita tributária) sobre o nível de atividade (produto) da economia brasileira, levando em consideração os possíveis efeitos dos choques da atividade econômica sobre as receitas fiscais do governo central, na matriz estrutural de modelos autorregressivos, a partir de uma análise de sensibilidade de parâmetros.

$\mathrm{O}$ artigo contém cinco seções, além desta introdução. A primeira seção traz uma discussão sobre o estado da arte a respeito do tema. A ideia é apresentar as limitações metodológicas das pesquisas que abordam o tema, especialmente para o caso brasileiro. A segunda seção discute a metodologia SVAR aplicada na pesquisa, expondo as etapas de construção da matriz estrutural. A seção três traz uma análise dos dados da pesquisa, sobretudo no que diz respeito ao processo gerador de dados. A quarta seção apresenta os resultados do artigo. Por fim, são apresentadas as considerações finais da pesquisa no intuito de identificar suas principais contribuições.

\section{MULTIPLICADORES FISCAIS: UMA ANÁLISE DA LITERATURA}

A popularização da análise de séries temporais na medição dos efeitos de choques fiscais parece não convergir a um consenso em relação aos resultados empíricos. Há um conjunto de fatores que podem justificar essa evidência conflitante. Por um lado, há o problema da escolha do número de variáveis endógenas, da estratégia de identificação, das características do país e até mesmo do período de estudo. Por outro, há o problema da metodologia; em particular, a escolha vai dos métodos mais convencionais - seja a 
decomposição de Choleski ou a identificação estrutural - a métodos mais avançados, como a abordagem bayesiana de restrição de sinais. Possivelmente, a metodologia por trás da mensuração dos multiplicadores fiscais desempenha papel central nos resultados.

$\mathrm{O}$ aspecto mais importante na medição dos efeitos de qualquer política é o procedimento usado para obter os chamados erros estruturais, em contraposição aos erros na sua forma reduzida. Esses erros são interpretados como choques exógenos e, portanto, as respostas do sistema a tais choques são legitimamente interpretadas como respostas reais. Ao contrário, o erro na forma reduzida em cada equação do sistema autorregressivo está correlacionado aos erros da outra equação, impedindo uma interpretação econômica desses choques, uma vez que não são exógenos. Assim, a credibilidade das funções impulso-resposta está relacionada à credibilidade dada ao procedimento de identificação utilizado.

Em função do horizonte de dados disponíveis, o conjunto mais natural de séries temporais para testar diferentes metodologias é o das séries macroeconômicas dos Estados Unidos. Com séries fiscais trimestrais oficiais e do PIB (e seus componentes) disponíveis a partir dos anos 1950, é possível contrastar os resultados encontrados por Blanchard e Perotti (2002) - que popularizaram o procedimento de identificação estrutural - com os de Mountford e Uhlig (2009) - que introduziram a abordagem de restrição de sinais nas análises de política fiscal.

A estratégia utilizada por Blanchard e Perotti (2002) para encontrar os erros estruturais foi calcular o efeito da atividade econômica sobre as receitas fiscais e, na sequência, a sua imposição sobre o modelo autorregressivo. Mountford e Uhlig (2009) identificam os erros estruturais através da abordagem de restrição de sinais, de modo que cada choque produz um comportamento pré-estabelecido nas variáveis do sistema nos primeiros trimestres. As principais conclusões de ambos divergem quanto às prescrições de política fiscal. Blanchard e Perotti (2002) sugerem que uma política de gastos é mais eficaz em afetar a atividade econômica, enquanto Mountford e Uhlig (2009) sustentam que uma política tributária, em termos de velocidade e impacto, teria mais eficácia sobre a produção.

A comparação dos resultados de Blanchard e Perotti (2002) e de Mountford e Uhlig (2009) dá uma clara ideia de que as funções impulso-resposta dependem fundamentalmente do método utilizado para derivar os erros estruturais. Na mesma linha da literatura internacional, não há um consenso sobre os efeitos reais da política fiscal brasileira.

Há um espaço na pesquisa para o caso brasileiro, ao passo que a evidência da literatura de vetores autorregressivos aplicada à política fiscal no Brasil varia entre respostas keynesianas tradicionais e casos com dinâmicas exatamente opostas.

Peres (2006, 2012), Peres e Ellery Jr. (2009) e Cavalcanti e Silva (2010) usam a identificação estrutural em modelos autorregressivos com três variáveis, na mesma linha que Blanchard e Perotti (2002). Correia e Oliveira (2013) estimam modelos 
estruturais com cinco variáveis endógenas em um conjunto de dados mensal. E, finalmente, a aplicação bayesiana de Mendonça, Medrano e Sachsida (2009), que aplica a metodologia em um modelo de seis variáveis. O modelo bayesiano de Mendonça, Medrano e Sachsida (2009) está incluso na discussão porque seus resultados são mais distintos em comparação ao grupo anterior de estudos que usam a identificação estrutural.

Os resultados apresentados por Peres (2006) e Peres e Ellery Jr. (2009) mostram que as funções impulso-resposta direcionam para um comportamento keynesiano, ou seja, uma resposta positiva ao choque de despesa e negativa ao choque tributário, sendo o choque de gastos mais forte que o choque tributário.

O modelo estrutural de Peres (2012) para o período 1994:1-2012:1 traz resultados que corroboram os apresentados por Peres (2006) e Peres e Ellery Jr. (2009). Cavalcanti e Silva (2010) também medem o efeito fiscal sobre a atividade econômica com uso da metodologia SVAR com três variáveis endógenas (despesas governamentais, receitas fiscais e produto). No entanto, seu principal objetivo é comparar as funções impulso-resposta de dois modelos, sendo a única diferença entre eles a presença da dívida pública como variável exógena. A análise de Cavalcanti e Silva está comprometida por estimativas imprecisas - grandes intervalos de confiança -, mas ainda assim, alguns resultados merecem destaque. Os efeitos dos gastos são mais fracos e menos persistentes no modelo com dívida. $\mathrm{O}$ modelo sem dívida mostra um efeito negativo para um dado choque fiscal, enquanto o modelo com dívida mostra um efeito positivo (para o mesmo tipo de choque).

Na mesma linha que Cavalcanti e Silva (2010), Correia e Oliveira (2013) incorporam a dívida no modelo autorregressivo, porém de maneira endógena. Os autores também comparam os resultados entre o modelo com dívida e o modelo sem dívida. O principal objetivo do trabalho foi avaliar o impacto da mudança nas regras fiscais no Brasil com a execução da Lei de Responsabilidade Fiscal (LRF) a partir de 2002. Foi incorporado ao modelo uma variável dummy para capturar o efeito da LRF. Correia e Oliveira (2013) utilizam os gastos governamentais, a atividade econômica, inflação e taxa de juros, além, obviamente, da própria dívida pública. As evidências sugerem que a inclusão da dívida faz com que o choque fiscal (via gastos) crie respostas mais persistentes e positivas quando se inclui a dívida. Esse resultado favorece a interpretação de que a modelagem de impulsos fiscais sem dívida pode introduzir um viés nas funções impulso-resposta ao superestimar os efeitos dos choques fiscais sobre a atividade econômica.

A análise de Mendonça, Medrano e Sachsida (2009) utiliza a abordagem de restrição de sinais nas funções impulso resposta. A estimativa do modelo com restrição de sinais é feita para o período 1997:1-2007:4 (dados trimestrais). O modelo contém seis variáveis endógenas - gastos públicos, receitas fiscais, taxa de juros, atividade econômica, inflação e consumo privado. Os resultados são opostos aos modelos de identificação estrutural, ou seja, a atividade econômica responde negativamente aos choques de gastos. 
Matheson e Pereira (2016), com dados das despesas e receitas primárias do governo central no período 1994:1-2014:1 (dados trimestrais), estimaram o multiplicador fiscal do gasto no Brasil ao nível de 0,5 , que pode ser considerado elevado para uma economia emergente, mas ainda abaixo das economias avançadas. Os resultados são superiores aos valores encontrados pela literatura, porém, os multiplicadores de receitas permaneceram praticamente estáveis.

Costa Junior, Cintado e Sampaio (2017) identificam o gasto público e o investimento público como os instrumentos fiscais mais efetivos na fase de contração da economia em momentos de crise, de modo que o multiplicador fiscal mais alto é associado às reduções no imposto sobre o consumo. Barros Neto e Correia (2020), levando em consideração eventos específicos, estimam o multiplicador fiscal dos gastos superior a um. Holland, Marçal e Prince (2020) não conseguiram identificar um impacto relevante dos estímulos fiscais sobre a atividade econômica, de modo que uma justificativa para a diminuição do efeito da política fiscal sobre o produto no Brasil é a combinação entre o elevado nível de gastos como proporção do PIB e uma tendência de alta das despesas públicas.

Mesmo que a literatura empírica brasileira forneça evidências de que os multiplicadores dos gastos públicos são positivos, existe a possibilidade de eles serem estatisticamente insignificantes. Ao mesmo tempo, a literatura deixa algumas lacunas em aberto que devem ser exploradas. Em primeiro lugar, os trabalhos anteriores realizaram testes de raiz unitária, porém, restringiram-se aos tradicionais testes, sem levar em consideração problemas de quebra estrutural (o que pode influenciar os resultados).

Em segundo lugar, existem diferenças na identificação dos modelos estruturais entre os trabalhos que tratam do caso brasileiro. Por exemplo, Peres $(2006,2012)$ segue o procedimento estabelecido por Blanchard e Perotti (2002) ao especificar a resposta das receitas fiscais aos choques na atividade econômica, assumindo que o governo não tem capacidade imediata para uma resposta discricionária dentro do mesmo trimestre. Já Cavalcanti e Silva (2010) e Correia e Oliveira (2013), ao contrário, identificaram seu modelo testando várias regras fiscais. Mesmo que uma regra fiscal possa ser interpretada como não sendo uma política discricionária, ela pressupõe uma grande capacidade, por parte do governo, de prever choques econômicos. Esta é uma suposição problemática a ser feita com relação aos eventos trimestrais, como expressam Blanchard e Perotti (2002).

As limitações metodológicas, bem como os procedimentos de especificação dos trabalhos apresentados, podem explicar a controvérsia em relação ao valor dos multiplicadores fiscais. Todavia, a análise dos determinantes dos multiplicadores fiscais pode fornecer respostas à tentativa de mensurá-los. Há um conjunto de elementos que devem ser levados em consideração no entendimento dos determinantes dos multiplicadores fiscais. 
No caso do Brasil, o aumento contínuo da carga tributária desde o início do processo de estabilização econômica na década de 1990 deve ser levado em consideração para os seus possíveis impactos sobre os multiplicadores fiscais: além de implicar que a economia brasileira, após o grande aumento da carga tributária, tivesse características bem diferentes da economia do início dos anos 1990 (no mínimo em relação a distribuição dos recursos entre o setor público e o setor privado), este movimento pode estar associado, ainda mais importante, à sensibilidade da arrecadação do governo diante dos ciclos econômicos, i.e. na carga tributária marginal da economia brasileira.

\section{METODOLOGIA}

Esta seção apresenta a metodologia utilizada na identificação do SVAR sob a hipótese de estabilidade e estacionariedade. A partir da matriz de variância-covariância do modelo autorregressivo em forma reduzida, é possível recuperar os parâmetros estruturais de correlações contemporâneas entre as variáveis endógenas. Como será detalhado adiante, para que o procedimento seja efetivado, é necessário impor valores (razoáveis) em alguns desses parâmetros - preferencialmente com argumentos sólidos de teoria econômica e/ou da própria relação entre as variáveis.

As relações contemporâneas são recuperadas do modelo autorregressivo na forma reduzida. Em (1), essas relações são tornadas explícitas através da matriz estrutural $A$ :

$$
\begin{gathered}
A x_{t}=A v+A \lambda(L) x_{t-1}+B \varepsilon_{t} \\
A u_{t}=B \varepsilon_{t}
\end{gathered}
$$

Em (1), $x$ é um vetor coluna de ordem $k$ das variáveis endógenas; $v$ é um vetor coluna de ordem $k$ com as constantes das equações (em alguns contextos, $v$ poderia ser expandido para representar uma matriz que agregue outros componentes determinísticos além da própria constante, como tendência, dummies sazonais ou quebra estruturais exógenas); $\lambda(L)$ é uma função polinomial em $t-1$, onde cada elemento é uma matriz $(k \times k) ; A$ descreve os efeitos contemporâneos, a matriz estrutural; $u_{t}$ é um vetor coluna, ruído branco de ordem $k$. O vetor $\varepsilon_{t}$, um ruído branco, possui variância unitária e sem correlação cruzada. A matriz $B$ representa os efeitos contemporâneos dos choques $\varepsilon_{t}$ sobre os choques na forma reduzida $u_{t}$. Assim, $B$ é uma matriz diagonal.

Considere o modelo com três variáveis como apresentado por Blanchard e Perotti (2002):

$$
x=\left[\begin{array}{l}
g \\
y \\
\tau
\end{array}\right]
$$

Em (2), $g$ representa as despesas governamentais em termos reais; $y$ é a medida de atividade econômica real e $\tau$ as receitas fiscais do governo. 
Uma possível estratégia de identificação da matriz estrutural é o método recursivo, que impõe um ordenamento sobre a ordem causal das variáveis do modelo autorregressivo. Nessa metodologia, é comum, em estudos empíricos de política fiscal com um vetor de variáveis endógenas igual ou semelhante a (2), que o gasto do governo seja a variável mais exógena (i.e. influencia contemporaneamente o produto e a receita tributária, mas não é influenciado contemporaneamente por nenhuma das variáveis); seguido pelo produto (que tem impacto contemporâneo sobre as receitas tributárias e sofre impacto dos gastos do governo) e, finalmente, pelas receitas tributárias (que não têm impacto sobre nenhuma variável, mas recebem impacto contemporâneo das anteriores). A equação (3) mostra uma representação da relação entre os termos de erro na forma reduzida e os termos de erro estruturais na ordenação recursiva:

$$
\begin{gathered}
A_{R} u_{t}=B \varepsilon_{t} \\
{\left[\begin{array}{ccc}
1 & 0 & 0 \\
-\alpha_{y g}^{R} & 1 & 0 \\
-\alpha_{\tau g}^{R} & -\alpha_{\tau y}^{R} & 1
\end{array}\right]\left[\begin{array}{c}
u_{t}^{g} \\
u_{t}^{y} \\
u_{t}^{\tau}
\end{array}\right]=B\left[\begin{array}{c}
\varepsilon_{t}^{g} \\
\varepsilon_{t}^{y} \\
\varepsilon_{t}^{\tau}
\end{array}\right]}
\end{gathered}
$$

Como exposto no parágrafo anterior, em (3) a ordem causal das variáveis implica que o impacto contemporâneo dos tributos sobre a atividade econômica seja igual a zero $\left(\alpha_{y \tau}^{R}=0\right)$, além da incapacidade de resposta imediata do governo ao que ocorre com a atividade econômica ou com suas próprias receitas $\left(\alpha_{y g}^{R}=\alpha_{y \tau}^{R}=0\right)$.

A hipótese $\alpha_{y \tau}=0$ parece ser problemática, não existindo nenhuma razão para supor que não haverá impactos contemporâneos na atividade econômica devido a choques nos tributos. No modelo recursivo, no entanto, não impor $\alpha_{y \tau}=0$ implica alterar a ordem de causalidade das variáveis, ou seja, impor o valor zero a algum parâmetro livre. Assumir que $\alpha_{y \tau}$ pode ser diferente de zero pode gerar a necessidade de impor, por exemplo, que $\alpha_{\tau y}$ seja zero.

Nenhuma das soluções acima é ideal. Blanchard e Perotti (2002) recorrem à identificação estrutural, utilizando informação externa (i.e. de outros trabalhos) sobre o valor de curto prazo da resposta das receitas tributárias a choques na atividade econômica, restringindo o valor de $\alpha_{\tau y}$ para um número diferente de zero. Peres (2006) calculou este parâmetro para a economia brasileira para o período 1994-2005, usando dados trimestrais. $\mathrm{O}$ autor encontrou um valor muito próximo aos próprios cálculos de Blanchard e Perotti (2002).

Com relação à imposição de que $\alpha_{g y}=\alpha_{g \tau}=0$, não há controvérsia na literatura. A restrição desses parâmetros está baseada no argumento institucional de que o governo não tem condições de responder contemporaneamente ao cenário econômico. Primeiro, principalmente porque os países ocidentais têm uma estrutura de decisões políticas que impede respostas rápidas por parte do executivo (em relação à frequência de dados 
trimestrais) devido às negociações com o poder legislativo. Segundo, mesmo que o executivo tenha o poder de responder de forma autônoma em alguma medida, não parece factível que ele tenha sequer conhecimento razoável do que ocorre no ambiente econômico no curto prazo, impedido uma resposta sistemática em virtude de variações no produto ou na receita tributária. Este segundo ponto parece ser ainda mais grave em países em desenvolvimento, cuja incerteza em relação aos dados parecer ser maior.

Finalmente, utilizando informações externas para estabelecer o parâmetro $\alpha_{\tau y} \mathrm{e}$ restringindo $\alpha_{g y}$ e $\alpha_{g \tau}$ a zero, torna-se possível estabelecer um modelo estrutural exatamente identificado. A matriz de variância-covariância na forma reduzida $\Sigma_{u}$ nos dá $6\left[=\left(k^{2}+k\right) / 2\right]$ parâmetros distintos que podem ser usados para identificar seis incógnitas: três na diagonal principal da matriz $B$ (desvio-padrão) e três da matriz estrutural ( $\alpha_{y g}, \alpha_{\tau g}$ e $\left.\alpha_{y \tau}\right)$. Em (4), o parâmetro $\alpha_{y \tau}$ - o impacto contemporâneo das receitas fiscais sobre a atividade econômica -, que foi restrito a zero na identificação recursiva, agora é um parâmetro livre:

$$
\begin{aligned}
& A_{S} u_{t}=B \varepsilon_{t} \\
& {\left[\begin{array}{ccc}
1 & 0 & 0 \\
-\alpha_{y g}^{S} & 1 & -\alpha_{y \tau}^{S} \\
-\alpha_{\tau g}^{S} & -\alpha_{\tau y}^{S} & 1
\end{array}\right]\left[\begin{array}{c}
u_{t}^{g} \\
u_{t}^{y} \\
u_{t}^{\tau}
\end{array}\right]=\left[\begin{array}{ccc}
\beta_{g g} & 0 & 0 \\
0 & \beta_{y y} & 0 \\
0 & 0 & \beta_{\tau \tau}
\end{array}\right]\left[\begin{array}{c}
\varepsilon_{t}^{g} \\
\varepsilon_{t}^{y} \\
\varepsilon_{t}^{\tau}
\end{array}\right]}
\end{aligned}
$$

Fazendo uso de informações externas sobre resposta das receitas tributárias ao produto, assim como de premissas institucionais sobre a capacidade do governo de responder contemporaneamente ao ambiente econômico, recupera-se os erros estruturais $\varepsilon_{t}$ (independentes entre si) que, como choques exógenos, podem ser utilizados na mensuração do impacto de cada variável no sistema através, por exemplo, da mensuração das funções impulso-resposta estruturais. O objetivo deste trabalho não é o cálculo das respostas dinâmicas dos choques fiscais, mas sim o estudo dos impactos contemporâneos. A análise de sensibilidade que será feita a seguir, no entanto, têm efeito importante sobre a forma das funções impulso-resposta: em muitos casos, o parâmetro contemporâneo da matriz estrutural parece dominar toda a dinâmica dos impactos dinâmicos de choques fiscais.

\section{ANÁLISE DOS DADOS}

Na etapa de organização dos dados, é importante destacar a grande diferença em relação à disponibilidade de séries macroeconômicas trimestrais no Brasil em comparação com os principais países da Organização para a Cooperação e Desenvolvimento Econômico (OCDE). Para esses últimos, o tamanho da amostra é maior que as amostras de países em desenvolvimento, como o Brasil. O Sistema de Contas Nacionais do Brasil (SCN) do Instituto Brasileiro de Geografia e Estatística (IBGE) disponibiliza dados trimestrais 
do PIB somente a partir do primeiro trimestre de 1995. Compare esse horizonte de tempo às séries temporais de dados macroeconômicos trimestrais para a economia dos EUA com mais de 200 observações, com dados a partir de 1950. Alguns outros países desenvolvidos (Reino Unido, Canadá, Austrália e Alemanha, por exemplo) têm séries temporais comparáveis desde a década de 1960.

Em relação ao estado das variáveis fiscais brasileiras, as limitações são bem maiores quando comparada aos dados das Contas Nacionais. Em primeiro lugar, as séries completas de despesas e receitas fiscais para o governo consolidado (governo federal, estadual e municipal) estão ausentes. Existem, no entanto, bons dados fiscais mensais para o governo central a partir de 1997. É possível então transformar esses dados mensais em dados trimestrais.

Para as receitas fiscais consolidadas, há uma série confiável a partir de Santos e Costa (2008) apenas para o período limitado de 1995:1-2007:4. Mesmo que houvesse uma fonte alternativa para os gastos consolidados, o modelo sofreria o problema dos graus de liberdade, o que resultaria em estimativas mais imprecisas. Provavelmente esta é a razão pela qual Peres $(2006,2012)$ e Ilzetzki (2011) também optaram por trabalhar com os dados do governo central em um cenário semelhante.

Dadas as limitações, é possível começar o exame dos dados reais. Primeiro, a medida da atividade econômica é o PIB, disponibilizado pelo SCN. As séries do PIB, tanto nominal quanto real, estão disponíveis a preços de 1995:1. Pode-se, com essas duas séries, derivar o deflator implícito e usá-lo para trazer também as variáveis fiscais a preços de 1995:1. As receitas e despesas do governo central são disponibilizadas pela STN, os valores reais e trimestrais podem ser construídos somando-se os valores mensais e aplicando o mesmo deflator do PIB implícito acumulado derivado da série do PIB. As séries foram ajustadas sazonalmente pelo método ARIMA - X12 e o período em análise começa em 1997:1 e termina em 2015:1, compreendendo três administrações presidenciais e cinco mandatos.

Um ponto de destaque em relação ao período de investigação, entre o primeiro trimestre de 1997 e o primeiro trimestre de 2015, é que o estudo respeita a consolidação de três ciclos fiscais no Brasil. Entre 1997 e 2015, Pires (2017) identifica três ciclos fiscais: i) o ciclo fiscal em 2002-03; ii) a política anticíclica de 2008-09; e iii) o ciclo recessivo a partir de 2014 com reversão da política fiscal em 2015. Dessa forma, o recorte temporal da pesquisa se justifica por compreender fases distintas na execução da política fiscal brasileira, uma vez que tal reversão da política fiscal em 2015, até a presente data, ainda não se consolidou.

\section{ANÁLISE DE RAIZ UNITÁRIA}

O diagnóstico do processo gerador de dados definirá a maneira como as séries temporais devem ser transformadas antes de serem inseridas no modelo empírico. A depender 
do diagnóstico dos testes de raiz unitária, portanto, as variáveis de fato que serão utilizadas no modelo VAR podem variar, o que pode gerar estimativas distintas dos parâmetros e das respostas dinâmicas, em última análise.

A Tabela 1 fornece três dos testes de raiz unitária mais tradicionais - Dickey Fuller aumentado (ADF), Phillips-Perron (PP) e Dickey-Fuller generalizado por mínimos quadrados (DF-GLS). Este último é um teste Dickey-Fuller modificado proposto por Elliot, Rothenberg e Stock (1996). O DF-GLS sofre uma transformação por mínimos quadrados generalizados antes da estimativa real da regressão por Dickey-Fuller, tornando possível o cálculo, endogenamente, do número ótimo de defasagens.

Tabela 1 - Teste de raiz unitária (ADF, PP e DF-GLS)

\begin{tabular}{lccccc}
\hline \multirow{2}{*}{ Variáveis } & DF-GLS & \multicolumn{2}{c}{ ADF } & \multicolumn{2}{c}{ PP } \\
\cline { 2 - 6 } & [estat-t] & $\rho$ & {$[$ estat-t] } & $\rho$ & {$[$ estat-t] } \\
\hline $\log [$ PIB] : tendência & {$[-1,261]$} & 0,858 & {$[-2,585]$} & 0,894 & {$[-2,215]$} \\
$\log [$ PIB] $:$ constante & {$[-0,648]$} & 0,998 & {$[-0,294]$} & 0,998 & {$[-0,293]$} \\
$\log [$ Receitas] : tendência & {$[-0,750]$} & 0,684 & {$[-2,488]$} & 0,494 & {$[-4,743]^{* * *}$} \\
$\log [$ Receitas] : constante & {$[0,244]$} & 0,963 & {$[-1,688]^{* *}$} & 0,958 & {$[-1,942]$} \\
$\log [$ Gastos]: tendência & {$[-3,050]^{*}$} & 0,619 & {$[-3,335]^{*}$} & 0,552 & {$[-4,466]^{* * *}$} \\
$\log [$ Gastos]: constante & {$[2,461]$} & 0,995 & {$[-0,324]$} & 0,995 & {$[-0,529]$} \\
\hline
\end{tabular}

Fonte: Elaboração própria.

Os testes ADF, PP e DF-GLS são realizados com as séries em logaritmo ajustadas sazonalmente. Em todos os casos, a hipótese nula é a de raiz unitária. Todas as variáveis se mostraram não-estacionárias, mas aplicando testes mais robustos, os resultados foram ambíguos para a presença de raiz unitária.

Uma fonte que pode invalidar a identificação do modelo estrutural é a presença potencial de quebra estrutural nos dados. Sabe-se que processos de raiz unitária e processos estacionários ao redor de tendência determinística podem se comportar de forma muito similar. Quebras estruturais tornam essa distinção ainda mais difícil pelo fato de que processos estacionários com quebra podem ser confundidos com processos não estacionários. Este fator aumenta a probabilidade de viés nas estatísticas dos testes tradicionais, que podem não rejeitar a hipótese nula, mesmo que esta seja falsa. Na tentativa de identificar a presença de quebra estrutural nas séries, foram levados em consideração testes de quebra estrutural como os de Zivot e Andrews (1992) e Clemente et al. (1998).

O teste desenvolvido por Zivot e Andrews (1992) é um teste de raiz unitária em que a localização da quebra é escolhida endogenamente por um algoritmo interno. A hipótese nula consiste em um processo raiz-unitária (e nenhuma quebra). A hipótese alternativa é que o processo gerador de dados seja estacionário ao redor de uma tendência 
determinística com uma quebra estrutural. Existem três formas de proceder o teste: i) a quebra estrutural ocorre na média (constante); ii) a quebra estrutural ocorre na tendência; e iii) a quebra estrutural ocorre na constante e na tendência.

O teste de Clemente, Montanés e Reyes (1998) também escolhe os pontos de quebra com um procedimento endógeno. Assim como no teste Zivot-Andrews, os períodos de quebras são escolhidos a partir da minimização da estatística $t$ do componente autorregressivo, isto é, os períodos das quebras são aqueles que geram estimativas do coeficiente menos favoráveis para a hipótese nula. Por outro lado, a distribuição da estatística $t$ é diferente da dos testes de raiz unitária tradicionais, com intervalos de confiança maiores. Ao contrário do teste anterior com uma quebra estrutural, o teste de Clemente, Montanés e Reyes (1998) tem como hipótese alternativa um processo gerador de dados que é estacionário ao redor da média.

Tabela 2 - Teste de quebra estrutural Zivot-Andrews (uma quebra estrutural)

\begin{tabular}{lcccccc}
\hline \multirow{2}{*}{ Variáveis } & Quebra & Data & {$[$ estat-t] } & \multicolumn{3}{c}{ Valores Críticos } \\
& & & & $\mathbf{1 \%}$ & $\mathbf{5 \%}$ & $\mathbf{1 0 \%}$ \\
\hline $\log [\mathrm{PIB}]$ & Constante & $(2006: 3)$ & {$[-3,567]$} & {$[-5,34]$} & {$[-4,80]$} & {$[-4,58]$} \\
$\log [\mathrm{PIB}]$ & Tendência & $(2012: 2)$ & {$[-2,970]$} & {$[-4,93]$} & {$[-4,42]$} & {$[-4,11]$} \\
$\log [\mathrm{PIB}]$ & Ambas & $(2009: 3)$ & {$[-3,193]$} & {$[-5,57]$} & {$[-5,08]$} & {$[-4,82]$} \\
$\log [$ Receitas $]$ & Constante & $(2012: 2)$ & {$[-3,592]$} & {$[-5,34]$} & {$[-4,80]$} & {$[-4,58]$} \\
$\log [$ Receitas $]$ & Tendência & $(2008: 1)$ & {$[-4,396]^{*}$} & {$[-4,93]$} & {$[-4,42]$} & {$[-4,11]$} \\
$\log [$ Receitas $]$ & Ambas & $(2008: 4)$ & {$[-4,598]$} & {$[-5,57]$} & {$[-5,08]$} & {$[-4,82]$} \\
$\log [$ Gastos $]$ & Constante & $(2005: 4)$ & {$[-5,359]^{* * *}$} & {$[-5,34]$} & {$[-4,80]$} & {$[-4,58]$} \\
$\log [$ Gastos $]$ & Tendência & $(1999: 4)$ & {$[-5,178]^{* * *}$} & {$[-4,93]$} & {$[-4,42]$} & {$[-4,11]$} \\
$\log [$ Gastos $]$ & Ambas & $(2004: 4)$ & {$[-5,469]^{* *}$} & {$[-5,57]$} & {$[-5,08]$} & {$[-4,82]$} \\
\hline
\end{tabular}

Fonte: Elaboração própria.

A Tabela 2 apresenta os resultados do teste Zivot-Andrews. Observa-se que o teste fornece pontos de quebra diferentes, a depender da hipótese alternativa específica, ou seja, a depender de se a quebra estrutural ocorre na constante da regressão, na tendência ou em ambas. No caso do produto, o teste não rejeita a hipótese de raiz unitária em nenhuma das hipóteses; pode-se rejeitar a hipótese de raiz unitária para as receitas quando ocorre quebra estrutural na tendência (a 10\% de significância); as despesas do governo têm a hipótese nula rejeitada, seja qual for a hipótese de localização da quebra estrutural, a $5 \%$ e $1 \%$ de significância.

A Tabela 3 apresenta o teste de quebra endógena de Clemente, Montanés e Reyes (1998). Uma característica do teste é que ele pode ser construído de acordo com dois procedimentos alternativos. Em primeiro lugar, há o método additive outlier (AO), que capta mudanças repentinas, e em segundo, o método innovative outlier (IO), 
que captura mudanças graduais. O primeiro método tem duas etapas: estima-se o resíduo da regressão das variáveis originais contra uma constante e duas variáveis dummy; na sequência, o resíduo é regredido contra seu próprio lag. O segundo método é um procedimento de uma etapa: a série de tempo é regredida contra seu próprio valor retardado, duas variáveis de pulso, duas variáveis dummy e $k$ lag's da primeira diferença das dummies. Teoricamente, não se pode esperar que ambos produzam os mesmos pontos de ruptura.

Tabela 3 - Teste de raiz unitária de Clemente, Montanés e Reyes (1998)

\begin{tabular}{lccccccc}
\hline \multirow{2}{*}{ Variáveis } & \multirow{2}{*}{ Tipo } & \multicolumn{2}{c}{$\mathbf{1}^{\mathbf{a}}$ quebra } & \multicolumn{2}{c}{ 2 $^{\mathbf{a}}$ quebra } & \multicolumn{2}{c}{ Raiz unitária } \\
\cline { 3 - 8 } & AO & $(2005: 1)$ & {$[10,06]^{* * *}$} & $(2008: 2)$ & {$[8,969]^{* * *}$} & 0,828 & {$[-2,803]$} \\
\hline \multirow{2}{*}{$\log [\mathrm{PIB}]$} & IO & $(2003: 3)$ & {$[3,715]^{* * *}$} & $(2008: 4)$ & {$[2,458]^{* *}$} & 0,920 & {$[-3,406]$} \\
& AO & $(2004: 2)$ & {$[13,20]^{* * *}$} & $(2010: 1)$ & {$[6,728]^{* * *}$} & 0,679 & {$[-3,961]$} \\
$\log [$ Receitas $]$ & IO & $(2003: 3)$ & {$[3,349]^{* * *}$} & $(2009: 2)$ & {$[2,827]^{* * *}$} & 0,742 & {$[-4,258]$} \\
& AO & $(2005: 1)$ & {$[12,17]^{* * *}$} & $(2009: 2)$ & {$[8,252]^{* * *}$} & 0,747 & {$[-3,369]$} \\
$\log [$ Gastos] & IO & $(2003: 2)$ & {$[2,707]^{* * *}$} & $(2008: 3)$ & {$[1,565]$} & 0,894 & {$[-2,451]$} \\
\hline
\end{tabular}

Fonte: Elaboração própria.

A Tabela 3 fornece as estatísticas de teste para os métodos AO e IO. Aproximadamente, é possível afirmar que as quebras estão localizadas mais ou menos perto de eventos econômicos: a primeira no período entre o ajuste fiscal de 2003 e o início do crescimento econômico da década; a segunda, durante ou após a crise financeira, que também marcou o fim do período de alto crescimento. Assim, duas rupturas parecem dar uma melhor imagem dos eventos que atingiram a economia brasileira durante a década de 2000. Apesar de as quebras estruturais serem significativas, em nenhum caso houve rejeição da hipótese de raiz unitária. O baixo poder dos testes de raiz unitária fica evidente quando se considera que a não rejeição da hipótese nula ocorre mesmo com coeficientes autorregressivos muito abaixo da unidade, principalmente no caso das variáveis fiscais. Como exemplo, o teste tipo IO para o log das receitas tem coeficiente autorregressivo de 0,68 e, ainda assim, o teste de Clemente, Montanés e Reyes (1998) não consegue rejeitar a hipótese de raiz unitária.

A existência de estimativas bem abaixo da unidade de muitos dos coeficientes autorregressivos, aliada ao fato de os testes não conseguirem rejeitar a hipótese nula de raiz unitária, é a razão pela qual ocorre uma escolha definitiva do processo gerador de dados nas três séries de tempo utilizadas neste trabalho. A estratégia foi a estimação de dois modelos autorregressivos: o primeiro supondo que as variáveis endógenas (gastos do governo, produto e receita tributária) são processos com tendência estocástica 
(cuja transformação requerida para a estimação em modelos VAR estáveis é a primeira diferença); e o segundo supondo que as variáveis endógenas são processos estacionários ao redor de tendências determinísticas, o que gera a necessidade de retirada da tendência antes da estimação.

\section{ANÁLISE DOS RESULTADOS}

De modo a levar em conta a evidência da ambiguidade em relação ao processo gerador de dados - como apontado no final da seção anterior -, nesta seção se apresentam os resultados da estimação de dois modelos SVAR: o primeiro em que se supõe que o vetor de variáveis endógenas é composto por séries com tendência estocástica (raiz unitária); e o segundo em que se supõe que o vetor é composto por séries estacionárias ao redor de tendências determinísticas.

Não obstante, os resultados mais importantes são aqueles relacionados à análise de sensibilidade quando se supõem diferentes valores para o parâmetro que captura o efeito contemporâneo do produto sobre as receitas fiscais, $\alpha_{\tau y}$. A construção de uma análise de sensibilidade é reforçada pelo fato de que Ilzetzki (2011) estimou um valor muito menor para a economia brasileira $\alpha_{\tau y}=0,75$ em contraste com o valor encontrado em Peres (2006) de $\alpha_{\tau y}=2$. Assim, a análise de sensibilidade realizará o estudo levando em conta um intervalo para o parâmetro $\alpha_{\tau y}$, ou seja, $0,5 \leq \alpha_{\tau y} \leq 3$. O valor de $\alpha_{\tau y}=2$, estimado por Peres (2006), está próximo à estimativa de Blanchard e Perotti (2002) para o mesmo parâmetro com dados da economia norte-americana. A análise de sensibilidade sobre o parâmetro $\alpha_{\tau y}$ é importante dada a relevância do parâmetro no processo de identificação estrutural e como consequência, inclusive, na construção de funções impulso-resposta. Tal procedimento permitirá descobrir o efeito das diferentes magnitudes do parâmetro $\alpha_{\tau y}$ nos resultados dos multiplicadores fiscais.

É importante destacar que a análise de sensibilidade sobre o parâmetro $\alpha_{\tau y}$ capta cenários de alterações na carga tributária, uma vez que quanto maior o tamanho dos tributos em relação à atividade econômica, maior será a sensibilidade dos tributos a flutuações no nível de atividade econômica.

A Tabela 4 exibe as estimativas dos parâmetros da matriz estrutural em (4) estimados por máxima verossimilhança para os dois blocos de modelos (tendência estocástica e tendência determinística). A observação dos resultados permite identificar uma correlação positiva entre os parâmetros $\alpha_{y g}$ e $\alpha_{\tau y}$ : quanto maior o impacto contemporâneo do produto sobre as receitas fiscais, maior o impacto dos gastos sobre o produto. Há uma relação inversa entre $\alpha_{\tau g}$ e $\alpha_{\tau y}$ : quanto maior o efeito contemporâneo do produto sobre as receitas fiscais, menor o impacto dos gastos sobre as receitas fiscais. Uma terceira 
importante relação encontrada é a correlação positiva entre $\alpha_{y \tau}$ e $\alpha_{\tau y}$ : quanto maior o choque do produto sobre as receitas fiscais, maior o efeito das receitas fiscais sobre o nível de atividade econômica.

Duas observações em relação à significância estatística dos parâmetros. Em primeiro lugar, o parâmetro $\alpha_{\tau g}$ permanece com significância estatística apenas para pequenos valores do parâmetro $\alpha_{\tau y}$, ou seja, $\alpha_{\tau y} \leq 1$. Em segundo, os parâmetros que medem o efeito contemporâneo das receitas fiscais sobre o produto são estatisticamente significantes para valores elevados de $\alpha_{\tau y}$, ou seja, $\alpha_{\tau y} \geq 2$

A literatura empírica brasileira sobre o tema aponta, de maneira geral, para um efeito keynesiano dos gastos sobre o produto, e os resultados apresentados aqui confirmam esse efeito keynesiano com relação ao multiplicador dos gastos públicos. Todavia, a análise de sensibilidade permite extrair alguns resultados no tocante às escolhas políticas envolvidas.

Tabela 4 - Análise de sensibilidade para o parâmetro $\alpha_{\tau y}$

\begin{tabular}{|c|c|c|c|c|c|}
\hline \multicolumn{6}{|c|}{ Tendência estocástica } \\
\hline & $\alpha_{\tau y}=\mathbf{0 , 5}$ & $\alpha_{\tau y}=0,75$ & $\alpha_{\tau y}=1,0$ & $\alpha_{\tau y}=2,0$ & $\alpha_{\tau y}=\mathbf{3 , 0}$ \\
\hline$\alpha_{y g}$ & $0,100(0,042)^{\star *}$ & $-0,108(0,043)^{\star *}$ & $-0,117(0,044)^{* * *}$ & $-0,153(0,050)^{* * *}$ & $-0,193(0,059)^{* * *}$ \\
\hline$\alpha_{\tau g}$ & $-0,375(0,140)^{\star * *}$ & $-0,351(0,140)^{\star *}$ & $-0,327(0,142)^{\star *}$ & $-0,230(0,153)$ & $-0,134(0,173)$ \\
\hline$\alpha_{\tau y}$ & $-0,5$ & $-0,75$ & -1 & -2 & -3 \\
\hline$\alpha_{y \tau}$ & $0,008(0,034)$ & $0,028(0,035)$ & $0,049(0,036)$ & $0,135(0,045)^{* * *}$ & $0,227(0,062)^{* * *}$ \\
\hline$\beta_{g g}$ & $0,028(0,002)^{\star * *}$ & $0,028(0,002)^{\star * *}$ & $0,028(0,002)^{* * *}$ & $0,028(0,002)^{\star * *}$ & $0,028(0,002)^{\star * *}$ \\
\hline$\beta_{y y}$ & $0,009(0,001)^{* * *}$ & $0,010(0,001)^{* * *}$ & $0,010(0,001)^{* * *}$ & $0,011(0,001)^{* * *}$ & $0,013(0,002)^{* * *}$ \\
\hline$\beta_{\tau \tau}$ & $0,033(0,003)^{* * *}$ & $0,033(0,003)^{* * *}$ & $0,034(0,003)^{* * *}$ & $0,036(0,003)^{* * *}$ & $0,041(0,003)^{* * *}$ \\
\hline \multicolumn{6}{|c|}{ Tendência determinística } \\
\hline & $\alpha_{\tau y}=0,5$ & $\alpha_{\tau y}=0,75$ & $\alpha_{\tau y}=1,0$ & $\alpha_{\tau y}=2,0$ & $\alpha_{\tau y}=3,0$ \\
\hline$\alpha_{y g}$ & $-0,089(0,041)^{\star *}$ & $-0,096(0,041)^{\star *}$ & $-0,104(0,042)^{\star \star}$ & $-0,134(0,474)^{\star * \star}$ & $-0,168(0,057)^{\star * *}$ \\
\hline$\alpha_{\tau g}$ & $-0,032(0,140)^{\star *}$ & $-0,300(0,140)^{\star *}$ & $-0,277(0,141)^{\star *}$ & $-0,187(0,151)$ & $-0,097(0,170)$ \\
\hline$\alpha_{\tau y}$ & $-0,5$ & $-0,75$ & -1 & -2 & -3 \\
\hline$\alpha_{y \tau}$ & $-0,002(0,033)$ & $0,017(0,034)$ & $0,037(0,035)$ & $0,121(0,043)^{* * *}$ & $0,212(0,060)^{* * *}$ \\
\hline$\beta_{g g}$ & $0,025(0,002)^{* * *}$ & $0,025(0,002)^{* * *}$ & $0,025(0,002)^{* * *}$ & $0,025(0,002)^{* * *}$ & $0,025(0,002)^{* * *}$ \\
\hline$\beta_{y y}$ & $0,008(0,001)^{* * *}$ & $0,008(0,001)^{\star * *}$ & $0,008(0,001)^{* * *}$ & $0,009(0,001)^{* * *}$ & $0,011(0,001)^{\star * *}$ \\
\hline$\beta_{\tau \tau}$ & $0,029(0,002)^{* * *}$ & $0,030(0,002)^{* * *}$ & $0,030(0,002)^{* * *}$ & $0,032(0,003)^{* * *}$ & $0,036(0,003)^{\star * *}$ \\
\hline
\end{tabular}

Fonte: Elaboração própria.

Os resultados sugerem um importante trade-off a respeito do orçamento público: políticas que alteram o efeito do produto sobre as receitas fiscais, ou seja, que proporcionam uma mudança em $\alpha_{\tau y}$, geram modificações nos multiplicadores fiscais. Assim, políticas que busquem ampliar $\alpha_{\tau y}$ implicarão em um aumento no efeito negativo das receitas sobre 
o produto. Em contrapartida, a mesma política que possibilita aumento em $\alpha_{\tau y}$ proporciona aumento no multiplicador dos gastos do governo sobre o nível de atividade econômica.

Enquanto há divergência em relação às prescrições de política fiscal ao se comparar os resultados de Blanchard e Perotti (2002) e Mountford e Uhlig (2009), os resultados apresentados aqui levam em consideração que os efeitos da política fiscal, seja via gastos ou por um choque tributário, dependerão do efeito contemporâneo do produto sobre as receitas fiscais $\left(\alpha_{\tau y}\right)$.

A discussão apresentada na pesquisa chama a atenção para a necessidade de estimar os multiplicadores fiscais levando em conta possíveis mudanças da carga tributária brasileira. Os resultados aqui apresentados sugerem que uma maior carga tributária marginal amplia os multiplicadores fiscais. A literatura empírica, no entanto, parece não discutir os impactos macroeconômicos das distorções sobre os multiplicadores da carga tributária.

De fato, a necessidade de mensuração dos multiplicadores fiscais nunca deve ser discutida isoladamente, mas sim associada às distorções do sistema tributário. No Brasil, o aumento da carga tributária ocorreu com a economia em moderada expansão; não é o objetivo deste trabalho discutir se tal expansão prejudicou ou ampliou o crescimento econômico observado nas últimas duas décadas. O importante, neste caso, é destacar que a intensidade dos choques fiscais (variações exógenas nos gastos do governo ou nas receitas tributárias) podem ser dependentes da carga tributária marginal do país, i.e. da sensibilidade das receitas em relação ao ciclo econômico. A eficácia da política fiscal é uma questão importante na discussão da capacidade do governo de estabilizar a economia através de instrumentos fiscais.

\section{CONCLUSÕES}

O objetivo deste artigo foi mensurar os impactos contemporâneos das variáveis fiscais (gasto do governo e receita tributária) sobre a economia brasileira - no âmbito da matriz estrutural de modelos SVAR -, levando em consideração a magnitude da sensibilidade das receitas fiscais em relação ao ciclo econômico, a partir de uma análise de sensibilidade dos parâmetros.

Os trabalhos que utilizam modelos VAR com identificação estrutural geralmente fornecem evidências de que os efeitos das variáveis fiscais sejam tipicamente keynesianos, embora alguns gerem resultados contraditórios. Ao mesmo tempo, a literatura deixa algumas lacunas em aberto que devem ser exploradas. Em primeiro lugar, a maior parte das pesquisas realiza os testes de raízes unitárias restringindo-se aos tradicionais testes, sem levar em consideração problemas de quebra estrutural, o que pode influenciar os resultados. Em segundo lugar, há problemas na identificação do modelo estrutural na maior parte dos trabalhos que tratam do caso brasileiro. 
As limitações metodológicas, bem como os procedimentos de especificação apontados na revisão da literatura, podem explicar a controvérsia em relação ao valor dos multiplicadores fiscais. Todavia, a análise dos determinantes dos multiplicadores fiscais pode fornecer respostas para a tentativa de mensurá-los. Há um conjunto de elementos que deve ser levado em consideração no entendimento dos determinantes dos efeitos fiscais sobre o nível de atividade.

A sensibilidade da arrecadação do governo no ciclo econômico (a magnitude do aumento das receitas tributárias na expansão econômica ou a redução, na recessão), que pode ter sido influenciada pela dinâmica da carga tributária do país desde a década de 1990, deve ser levada em consideração para avaliar seus possíveis impactos sobre os efeitos das variáveis fiscais sobre a atividade econômica. Este é o principal resultado da análise de sensibilidade de parâmetros exibido na Tabela 4, e a conclusão não muda por conta da suposição do processo gerador de dados: independentemente de se as variáveis endógenas do modelo autorregressivo são raízes unitárias ou estacionárias ao redor de tendências determinísticas, os resultados mostram que uma maior sensibilidade de arrecadação tributária diante de mudanças de curto prazo no nível de atividade está positivamente correlacionada a uma maior eficácia da política fiscal (i.e. magnitude dos multiplicadores de gasto e tributos).

Nesse sentido, a principal contribuição deste artigo é introduzir, junto à literatura empírica aplicada ao caso brasileiro, especificamente em séries de tempo, a discussão da relação entre a sensibilidade da arrecadação aos movimentos de curto prazo do nível de atividade e os multiplicadores fiscais. Particularmente, a literatura de política fiscal em modelos VAR estruturais tem muito a ganhar com pesquisas futuras que procurem estimar a tendência recente dessa sensibilidade e, consequentemente, dos seus efeitos sobre multiplicadores fiscais. $\mathrm{O}$ desenho da carga tributária brasileira, também com relação à influência sobre essa sensibilidade dos tributos aos ciclos de atividade, parece ser um tema correlacionado. Por fim, se a política fiscal pode estar influenciando a magnitude do impacto que variáveis fiscais têm sobre o nível de atividade no curto prazo, torna-se necessário discutir (paralelamente) sobre a desejabilidade de se ter altos ou baixos multiplicadores fiscais.

\section{REFERÊNCIAS}

BARROS NETO, G. S.; CORREIA, F. M. Uma contribuição para as estimativas de multiplicadores fiscais no Brasil: Análise de intervenção em modelos VAR Ampliados por Dummies. Revista Brasileira de Economia, v. 74, n. 3, p. 235-254, 2020.

BLANCHARD, O.; PEROTTI, R. An empirical characterization of the dynamic effects of changes in government spending and taxes on output. The Quarterly Journal of Economics, v. 117, n. 4, p. 1329-1368, 2002. 
CAVAlCANTI, M. A. F. H.; SILVA, N. L. C. Dívida pública, política fiscal e nível de atividade: uma abordagem VAR para o Brasil no período 1995-2008. Economia Aplicada, v. 14, n. 4, p. 391-418, 2010.

CLEMENTE, J.; MONTANÉS, A.; Reyes, M. Testing for unit root in variables with a double change in the mean. Economic letters, v. 59, n. 2, p. 175-182, 1998.

CORREIA, F. M.; OLIVEIRA, V. Reversão cíclica da política fiscal brasileira e a lei de responsabilidade fiscal. Caderno de Finanças Públicas, v. 21, n. 1, p. 1-28, 2013.

COSTA JUNIOR, C. J.; CINTADO, A. C. G.; SAMPAIO, A. V. Post-2008 Brazilian fiscal policy: an interpretation through the analysis of fiscal multipliers. Estudos Econômicos, v. 47, n. 1, p. 93-124, 2017.

ELLIOT, G.; ROTHENBERG, T. J.; STOCK, J. H. Efficient tests for an auto-regressive unit root. Econometrica, v. 64, n. 4, p. 813-836, 1996.

HOLLAND, M.; MARÇAL, E.; PRINCE, D. Is fiscal policy effective in Brazil? An empirical analysis. The Quarterly Review of Economics and Finance, v. 75, p. 40-52, 2020.

ILZETZKI, E. Fiscal policy and debt dynamics in developing countries. World Bank Policy Research Paper, n. 5666, 2011.

MATHESON, T.; PEREIRA, J. Fiscal multipliers for Brazil. IMF Working Paper, n. 16/79, 2016.

MENDONÇA, M. J.; MEDRANO, L. A.; SACHSIDA, A. Avaliando efeitos da política fiscal no Brasil: resultados de um procedimento de identificação agnóstica. Texto para Discussão, IPEA, Brasília, n. 1377, 2009.

MOUNTFORD, A.; UHLIG, H. What are the effects of fiscal policy shocks? Journal of Applied Econometrics, v. 24, n. 6, p. 960-992, 2009.

PERES, M. A. F. Dinâmica dos choques fiscais no Brasil. 2012. Tese (doutorado em Economia) Departamento de Economia, Universidade de Brasília, Brasília, DF, 2012.

PERES, M. A. F. Efeitos dinâmicos da política fiscal sobre a atividade econômica: um estudo para o caso brasileiro. 2006. Dissertação (mestrado em Economia) - Departamento de Economia, Universidade de Brasília, Brasília, DF, 2006.

PERES, M. A. F., ELLERY JUNIOR, R. G. Efeitos dinâmicos dos choques fiscais do governo central no PIB do Brasil. Pesquisa e Planejamento Econômico, v. 39, n. 12, p. 159-206, 2009.

PIRES, M. Política fiscal e ciclos econômicos: teoria e a experiência recente. São Paulo: GEN Atlas, 2017.

SANTOS, C. H.; COSTA, F. R. Uma metodologia de estimação da carga tributária bruta brasileira em bases trimestrais. Economia Aplicada, v. 12, n. 4, p. 581-606, 2008.

ZIVOT, E.; ANDREWS, D. W. K. Further evidence on the great crash, the oil-price shock, and unit-root hypothesis. Journal of Business and Economics Statistics, v. 10, n. 3, p. 251-270, 1992. 